\title{
ATUAÇÃO DA FISIOTERAPIA NA REDUÇÃO DO LINFEDEMA NA PACIENTE MASTECTOMIZADA: UMA REVISÃO INTEGRATIVA
}

\author{
ACTION OF PHYSIOTHERAPY IN REDUCING LYMPHEDEMA IN MASTECTOMIZED PATIENTS: \\ AN INTEGRATIVE REVIEW
}

DOI: $10.16891 / 2317-434 X . v 10 . e 1 . a 2022 \cdot p p 1247-1250$

Recebido em: 10.11 .2021 | Aceito em: 10.11.2021

\begin{abstract}
Cícera Alexsandra Nascimento da Silva, Cicero Jefferson Martins Caetano, Larissa Gonçalves da Silva e Rafaela Macêdo Feitosa
\end{abstract}

Centro Universitário Doutor Leão Sampaio

\section{RESUMO}

A mastectomia é um procedimento cirúrgico conservador que consiste na retirada de uma das mamas ou ambas, com intuito de promover a ressecção do tumor ou das células neoplásicas adjacentes. Entretanto, trás consigo o linfedema; uma complicação tardia e bastante comum em decorrência dessa abordagem. As condutas fisioterapêuticas objetivam reduzir o volume do membro e os sintomas provenientes do linfedema, devolvendo qualidade de vida e funcionalidade sobre o membro acometido. Esse trabalho objetivou analisar as abordagens fisioterapêuticas mais eficazes e utilizadas para a redução do linfedema em pacientes pós-mastectomizadas. A presente pesquisa se caracteriza como uma revisão integrativa, realizada entre Setembro e Outubro de 2021 , a partir do levantamento de artigos científicos publicados nos últimos 5 anos, por meio das revistas eletrônicas especializadas PUBMED, BVS e PEDro utilizando como descritores: mastectomy, lymphedema e physiotherapy, através do operador boleano AND. Selecionou-se 6 artigos e os mesmos demostraram que entre as principais condutas fisioterapêuticas realizadas para resolução do quadro, estão incluídos a terapia descongestiva complexa, enfaixamento em multicamadas, drenagem linfática manual, e compressão pneumática intermitente. Todas as terapias se mostraram eficazes quando utilizadas individualmente ou em associação, demostrando melhora e grande influência na redução do linfedema e circunferência do braço edemasiado. Diante da leitura dos estudos, conclui-se que a atuação da fisioterapia é fundamental no processo de reabilitação nas pacientes mastectomizadas; visto que suas condutas visam melhorar a qualidade de vida, reduzir quadro álgico, edema e limitações para as AVD's, e devolver a funcionalidade as pacientes.

Palavras-chave: Linfedema; Fisioterapia; Mastectomia.

\section{ABSTRACT}

Mastectomy is a conservative surgical procedure that consists of removing one of the breasts or both, in order to promote the resection of the tumor or adjacent neoplastic cells. However, it brings lymphedema with it; a late and quite common complication as a result of this approach. Physiotherapeutic approaches aim to reduce limb volume and lymphedema-related symptoms, restoring quality of life and functionality to the affected limb. This study analyze the most effective physical therapy approaches used to reduce lymphedema in post-mastectomized patients. This research is characterized as an integrative review, carried out between September and October 2021, based on a survey of scientific articles published in the last 5 years, through the specialized electronic journals PUBMED, BVS and PEDro using as descriptors: mastectomy, lymphedema and physiotherapy, through the Boolean operator AND. Six articles were selected and they showed that among the main physical therapy procedures performed to resolve the condition, complex decongestive therapy, multilayer bandaging, manual lymphatic drainage, and intermittent pneumatic compression are included. All therapies proved to be effective when used individually or in combination, showing improvement and great influence on the reduction of lymphedema and circumference of the edematous arm. After reading the studies, it is concluded that the role of physiotherapy is essential in the rehabilitation process of mastectomized patients; as their conducts aim to improve quality of life, reduce pain, edema and limitations for ADLs, and restore functionality to patients.

Keyword: Lymphedema; Physiotherapy; Mastectomy. 


\section{V.10 N.1 (2022) ISSN: 2317-434X}

\section{INTRODUÇÃO}

O câncer de mama é um grupo heterogêneo de doenças, de caráter multifatorial causada pela multiplicação desordenada de células anormais do tecido mamário, com potencial de invadir outros órgãos. Acomete principalmente o público feminino, correspondendo atualmente por cerca de $28 \%$ dos casos de câncer neste público, com estimativa para o ano de 2021 cerca de 66.280 novos casos (INCA; 2021).

O tratamento antineoplásico quando realizado precocemente tem por finalidade favorecer as chances de cura do paciente. Podendo contar com radioterapia, quimioterapia, hormonioterapia, cirurgias conservadoras, reconstrução mamária e mastectomia; sendo esta última bastante utilizada para a eliminação do tumor. Caracterizase como uma conduta habitual nas fases iniciais do câncer e tem por objetivo a retirada das mamas, com intuito de promover a ressecção total do tumor ou das células neoplásicas adjacentes (BRASIL, 2021).

Dentre todas as repercussões ocasionadas pela mastectomia, o linfedema é uma das principais complicações. Ocorre de forma tardia e se caracteriza como um estado crônico do acúmulo de líquido linfático ocasionado pela cirurgia oncológica; frequentemente provocado pela remoção de linfonodos durante o procedimento. Alterando significativamente a capacidade funcional e qualidade de vida dessas pacientes (MARCHITO et al.,2019).

A Fisioterapia Onco-Funcional tem por finalidade melhorar a qualidade de vida e promover a independência funcional das pacientes, tratando as alterações cinéticofuncionais ocasionados pelo processo patológico e tratamento oncológico. Busca manter, preservar e restaurar a funcionalidade dos órgãos e tecidos acometidos através de técnicas e condutas específicas de forma individualizada e direcionada, respeitando o estado geral da paciente (VIANA, 2016).

Contudo, as condutas fisioterapêuticas objetivam reduzir o volume do membro e os sintomas provenientes do linfedema, devolvendo qualidade de vida e funcionalidade sobre o membro acometido. Fazendo-se necessária a realização de técnicas e condutas específicas que comprovam sua eficácia no tratamento dessa complicação (TANTAWY et al.,2019).
Dessa forma, este estudo foi realizado com objetivo de analisar as abordagens fisioterapêuticas mais eficazes e utilizadas para a redução do linfedema em pacientes pós-mastectomizadas.

\section{MÉTODO}

A presente pesquisa se trata de um estudo de revisão integrativa com abordagem descritiva. A qual foi realizada nas bibliotecas virtuais da National Center for Biotechnology Information PUBMED (Mesh), biblioteca virtual em saúde BVS (Desc), banco de dados PEDRO, Physiotherapy Evidence Database, utilizando os descritores mastectomy, lymphedema e physiotherapy, com o operador booleano AND. O período de realização ocorreu entre os meses de Setembro e Outubro de 2021, considerando elegíveis os estudos publicados em periódicos a partir de 2016; nos idiomas inglês, português e espanhol; do tipo ensaio clínico randomizado.

Dessa forma, foram incluídos na pesquisa estudos disponibilizados na integra que apresentaram os protocolos completos de tratamento fisioterapêutico para a redução do linfedema; contendo dados da amostra, períodos e resultados da realização, com a população tratando-se de mulheres pós-mastectomizadas. Sendo exclusos, artigos incompletos, que não apresentaram protocolo de tratamento completo, artigos duplicados, e de revisão de literatura.

Os estudos selecionados foram organizados em tabela modelo PICOT e analisados de forma criteriosa e esmiuçada a partir da leitura extenuada dos pesquisadores, seguindo a todos os critérios metodológicos.

\section{RESULTADOS E DISCUSSÃO}

Ao todo foram encontrados 157 artigos nas bases de dados e após o processo de seleção supracitado anteriormente, 6 artigos foram incluídos para a presente revisão. A seguir, apresenta-se tabela com breve resumo sobre os artigos selecionados para apresentação dos dados obtidos, sendo distribuídos em categorias com informações relevantes em relação aos efeitos da fisioterapia na redução do linfedema em pacientes pósmastectomizadas. 
V.10 N.1 (2022) ISSN: $2317-434 X$

Tabela 1. Apresentação dos resultados sobre os artigos selecionados para o estudo.

\begin{tabular}{|c|c|c|}
\hline TÍTULO & $\begin{array}{c}\text { AUTOR/ANO } \\
\text { TIPO DE ESTUDO }\end{array}$ & INTERVENÇÃO \\
\hline $\begin{array}{l}\text { Complex decongestive therapy } \\
\text { improves upper limb function in } \\
\text { patients with breast cancer-related } \\
\text { lymphedema. }\end{array}$ & $\begin{array}{l}\text { OZCAN, et al. } \\
2018 . \\
\text { Ensaio } \quad \text { clínico } \\
\text { randomizado }\end{array}$ & $\begin{array}{l}\text { Todos os pacientes foram submetidos ao programa de fase } 1 \text { do CDT, } \\
\text { incluindo cuidados meticulosos com a pele, drenagem linfática manual, } \\
\text { exercícios corretivos e bandagens compressivas. Todos os pacientes } \\
\text { receberam DLM cinco vezes por semana, durante } 3 \text { semanas. Na (fase 2) do } \\
\text { CDT foi orientado ao paciente e familiar para a fase de manutenção com } \\
\text { enfaixamento em várias camadas e técnicas de automassagem. }\end{array}$ \\
\hline $\begin{array}{l}\text { Comparative study between the } \\
\text { effects of Kinesio bandage and } \\
\text { pressure garment on secondary } \\
\text { upper extremity lymphedema and } \\
\text { quality of life after mastectomy: a } \\
\text { randomized clinical trial. }\end{array}$ & $\begin{array}{l}\text { TANTAW, et. al. } \\
2019 \\
\text { Ensaio clínico } \\
\text { randomizado }\end{array}$ & $\begin{array}{l}\text { Os participantes foram distribuídos aleatoriamente em } 2 \text { grupos. O grupo KT } \\
\text { recebeu a aplicação de bandagem Kinesio duas vezes por semana. O grupo } \\
\text { PG recebeu vestimenta de pressão }(20-60 \mathrm{~mm} \mathrm{Hg}) \text { por pelo menos } 15 \text { a } 18 \\
\text { horas por dia durante } 3 \text { semanas. Ambos os grupos receberam um programa } \\
\text { de exercícios em casa na forma de exercícios de amplitude de movimento, } \\
\text { caminhada na parede e alongamento da bengala. }\end{array}$ \\
\hline $\begin{array}{l}\text { A quasi-randomized clinical trial: } \\
\text { virtual reality versus proprioceptive } \\
\text { neuromuscular facilitation for post- } \\
\text { mastectomy lymphedema. }\end{array}$ & $\begin{array}{l}\text { ATEF, et. al. } \\
2020 \\
\text { Ensaio clínico quase } \\
\text { randomizado }\end{array}$ & $\begin{array}{l}30 \text { pacientes, das quais } 15 \text { pacientes realizaram exercícios baseados em } \\
\text { realidade virtual, bem como drenagem linfática manual, compressão } \\
\text { pneumática e programas domésticos, enquanto as outras } 15 \text { pacientes } \\
\text { realizaram FNP, bem como drenagem linfática manual, compressão } \\
\text { pneumática e programas domiciliares. }\end{array}$ \\
\hline $\begin{array}{l}\text { Efficacy of self-administered } \\
\text { complex decongestive therapy in } \\
\text { breast cancer-related lymphedema: a } \\
\text { single-blind, randomized clinical } \\
\text { trial. }\end{array}$ & $\begin{array}{l}\text { LIGABUE, et. al } \\
2019 . \\
\text { Ensaio clínico } \\
\text { randomizado cego de } \\
\text { grupo paralelo }\end{array}$ & $\begin{array}{l}\text { O grupo controle recebeu apenas os cuidados habituais fornecidos na alta } \\
\text { após a CDT, que consiste em um briefing ponto a ponto e discussão do } \\
\text { folheto. O grupo experimental recebeu o curso saCDT. O programa de cada } \\
\text { uma das } 10 \text { reuniões foi padronizado em termos de tópicos, demonstrações } \\
\text { práticas, ensaios e tempo de discussão com os pacientes. }\end{array}$ \\
\hline $\begin{array}{l}\text { Low-intensity resistance training } \\
\text { and compression garment in the } \\
\text { management of breast cancer-related } \\
\text { lymphedema: a single-blind } \\
\text { randomized clinical trial. }\end{array}$ & $\begin{array}{l}\text { Omaret al., } \\
2020 \\
\text { Ensaio } \\
\text { randomizado } \\
\text { cego. }\end{array}$ & $\begin{array}{l}\text { Um total de } 60 \text { mulheres com BCRL unilateral foram aleatoriamente } \\
\text { designadas para exercícios de resistência de baixa intensidade (grupo Rex, } \mathrm{n} \\
=30 \text { ) ou exercícios e roupa de compressão (grupo Rex-Com, } \mathrm{n}=30 \text { ). Ambos } \\
\text { os grupos participam de um programa de exercícios composto de } 10-12 \\
\text { repetições de } 50 \text { a } 60 \% \text { de uma repetição máxima (IRM), três vezes por } \\
\text { semana, durante } 8 \text { semanas. }\end{array}$ \\
\hline $\begin{array}{l}\text { Efficacy of four types of bandages } \\
\text { and kinesiotape for the treatment of } \\
\text { breast cancer-related lymphedema: a } \\
\text { randomized, single-blind clinical } \\
\text { trial. }\end{array}$ & $\begin{array}{lr}\text { TORRES } & \text { Lacomba } \\
\text { María, et al. } & \\
2020 & \\
\text { Ensaio } & \text { clínico } \\
\text { randomizado } & \text { simples- } \\
\text { cego } & \end{array}$ & $\begin{array}{l}\text { Os participantes foram randomizados em cinco grupos }(n=30) \text {. Todas as } \\
\text { mulheres receberam uma fase intensiva de fisioterapia descongestiva } \\
\text { complexa, incluindo drenagem linfática manual, terapia de compressão } \\
\text { pneumática, educação terapêutica, exercícios terapêuticos ativos e } \\
\text { enfaixamento. A única diferença entre os grupos foi a bandagem ou fita } \\
\text { aplicada. }\end{array}$ \\
\hline
\end{tabular}

FONTE: Dados da pesquisa (2021).

O ensaio clínico de Ozcan e colaboradores (2018) realizado em 2 fases, fazendo uso da Terapia Descongestiva Complexa, enaltece uma redução estatisticamente significativa nos escores da Escala Visual Analógica (VAS) de dor e peso e melhora da mobilidade do ombro entre os membros superiores com linfedema após a Terapia Descongestiva Complexa. Corroborando com o estudo de Torres et al. (2020), realizado com 150 mulheres, utilizando também a CDT em associação a bandagens de multicamadas, demonstrando uma diminuição significativa dos sintomas em todas as pacientes após as intervenções.
Entretanto, o estudo de Ligabue et al. (2019), um ensaio clínico randomizado, cego, de grupo paralelo, realizado em 4 semanas, utilizando a CDT autoadministrada no grupo experimental, não apresentou diferença significativa entre o grupo controle; encontrando uma grande variabilidade entre os sujeitos em ambos os grupos, tanto em termos de dor quanto de assimetria de braço.

Os estudos de Tantawy et al. (2019), e Omaret et al. (2020), trouxeram muitas semelhanças, principalmente no protocolo de tratamento e na amostra; porém o primeiro estudo foi realizado utilizando a terapia de Kinesio taping 


\section{V.10 N.1 (2022) ISSN: 2317-434X}

e a vestimenta de pressão, já o segundo estudo realizou exercícios de resistência de baixa intensidade e ou exercícios com roupa de compressão. Ressaltando que ambos os estudos obtiveram resultados satisfatórios para redução do linfedema e seus sintomas secundários, corroborando com o presente estudo; o qual demonstra eficácia da atuação da fisioterapia sobre a patologia em questão.

Outro estudo comparativo realizado por Atefet et al. (2020), abordou a terapia de realidade virtual versus facilitação neuromuscular proprioceptiva em 30 pacientes com linfedema pós-mastectomia unilateral; atingindo como resultado a diminuição expressiva do volume excessivo do braço tanto no grupo que recebeu a terapia de realidade virtual, quanto no grupo de facilitação neuromuscular proprioceptiva, propiciando um efeito terapêutico benéfico no edema em pacientes com linfedema pós-mastectomia unilateral. Salvo que a realidade virtual foi considerada superior à facilitação neuromuscular proprioceptiva na motivação dos pacientes e no fornecimento de feedback visual.

\section{CONSIDERAÇÕES FINAIS}

Conclui-se que a atuação da fisioterapia é fundamental no processo de reabilitação nas pacientes mastectomizadas; visto que suas condutas visam melhorar a qualidade de vida, reduzir as limitações na realização de atividades diárias e relações pessoais, devolvendo a funcionalidade e o bem-estar as pacientes. Reduzindo bastante seu quadro álgico, edema e limitação para as AVD's, através de recursos terapêuticos manuais e ou mecânicos.

\section{REFERÊNCIAS}

ATEF, Doaa et al. A quasi-randomized clinical trial: virtual reality versus proprioceptive neuromuscular facilitation for postmastectomy lymphedema. Journal of the Egyptian National Cancer Institute, v. 32, p. 1-9, 2020.

BRASIL, www.gov.br < Acessado em: 14 de setembro de 2021. Disponível em: https://www.gov.br/saude/ptbr/assuntos/saude-de-a-a-z/c/cancer-de-mama. 19/04/2021.

INCA, www.inca.gov.br <Acessado em: 14 de setembro de 2021. Disponível em: https://www.inca.gov.br/tiposde-cancer/cancer-de-mama. 02/09/2021.

LIGABUE, M. B. et al. Efficacy of self-administered complex decongestive therapy on breast cancer-related lymphedema: a single-blind randomized controlled trial. Breast cancer research and treatment, v. 175, n. 1, p. 191-201, 2019.

MARCHITO, Liz de Oliveira et. al. Prevenção e Cuidado do Linfedema após Câncer de Mama: Entendimento e Adesão às Orientações Fisioterapêuticas. Revista Brasileira de Cancerologia. v. 65, n. 1, 2019.

OMAR, Mohammed TA et al. Low-Intensity Resistance Training and Compression Garment in the Management of Breast Cancer-Related Lymphedema: Single-Blinded Randomized Controlled Trial. Journal of Cancer
Education, v. 35, n. 6, p. 1101-1110, 2020.

SEZGIN OZCAN, Didem et al. Complex decongestive therapy enhances upper limb functions in patients with breast cancer-related lymphedema. Lymphatic research and biology, v. 16, n. 5, p. 446-452, 2018. Disponível em: <https://www.liebertpub.com/doi/10.1089/lrb.2017.0061 ?url_ver=Z39.88-

$2003 \&$ rfr_id=ori:rid:crossref.org\&rfr_dat $=$ cr_pub $\% 20 \%$ 200pubmed>. Acessado em: 20 set. 2021.

TANTAWY, Sayed A et al. Comparative Study Between the Effects of Kinesio Taping and Pressure Garment on Secondary Upper Extremity Lymphedema and Quality of Life Following Mastectomy: A Randomized Controlled Trial. Integrative Cancer Therapies, v. 18, 2019. Disponível em: <https://www.ncbi.nlm.nih.gov/pmc/articles/PMC650997 4/>. Acessado em: 20 set. 2021.

TORRES-LACOMBA, María et al. Effectiveness of four types of bandages and kinesio-tape for treating breastcancer-related lymphoedema: a randomized, single-blind, clinical trial. Clin Rehabil, v. 34, n. 9, p. 1230-1241, 2020.

VIANA, B. L. A, Fisioterapia Oncológica In: VIEIRA, S. C, Oncologia básica para profissionais de saúde, 1. ed, Udufpi: Teresina, 2016. p. 49-54.

v. 6, n. 2, p. e13009, 2019. 